\title{
HER2 Status of Gastric Carcinoma and Corresponding Lymph Node Metastasis
}

\author{
Fabio Pagni • Stefano Zannella • Susanna Ronchi • \\ Cristina Garanzini • Biagio Eugenio Leone
}

Received: 4 November 2011 / Accepted: 9 August 2012

(C) Arányi Lajos Foundation 2012

\begin{abstract}
Our goal is to verify HER2 status variability between primary tumor and metastatic site. Our second intention is to identify the most reliable criteria for pathological HER2 status assessment in gastric cancer node metastases since, at present, there is not a validated standard. 3 independent pathologists evaluated HER2 immunohistochemical and gene status (for IHC $2+$ cases) in 34 gastric carcinoma metastatic lymph nodes and in their corresponding primary tumors. For primary gastric cancers, we followed the current HER2 assessment guidelines and for nodal metastases, we applied two immunohistochemical scoring systems with different cut-offs. The immunohistochemical inter-pathologists mean agreement was $71.4 \%(\kappa=0.45)$; a final score for each case was defined after collegial revision. By applying the two immunohistochemical criteria, we found 2 discordant cases, which can imply different pathological management. Moreover, a significantly different HER2 status between lymph node metastasis and primary tumor was obtained in 4 cases (concordance ratio $87.5 \%$ ). None of the patients would have undergone a different therapeutic pathway despite the scoring method applied. On the other hand we also detected a subset of patients who could have their therapeutic management changed, according to the differences between HER2 status in lymph nodes metastases and primary tumor.
\end{abstract}

Keywords Gastric cancer · Metastasis · HER2 $\cdot$ IHC $\cdot$ HER2 status scoring system

F. Pagni · S. Zannella · S. Ronchi · C. Garanzini · B. E. Leone Department of Pathology, Desio Hospital,

Desio, Italy

F. Pagni $(\bowtie) \cdot$ S. Zannella $\cdot$ S. Ronchi $\cdot$ C. Garanzini $\cdot$

B. E. Leone

University Milano Bicocca,

Milan, Italy

e-mail: petala.83@tiscali.it

\section{Introduction}

Lymph node metastasis is an early event in gastric carcinoma natural history. Sometimes, regional or distant node metastasis is the first sign of the disease. Where eligible, in metastatic patients, selective inhibitors of Human Epidermal growth factor Receptor 2 (HER2) had been added to conventional adjuvant and palliative chemotherapy regimens, which consist in epirubicin, cisplatin and 5fluorouracil [1]. Nowadays, many groups are also evaluating the possible role of HER2 inhibitors in neoadjuvant regimens. Eligibility criteria are defined by HER2 positive immunohistochemistry (IHC) or gene amplification in tumor cells, generally evaluated on endoscopic biopsies or surgical specimens, but not on node metastases. HER2 status in lymph node metastases is usually scored in patients with nodal disease recurrence or in inoperable cases, but not in lymphadenectomies performed during gastrectomy. The main aim of this study is to clarify HER2 status variability between primary tumor and its nodal metastasis. We also want to investigate criteria to define HER2 positivity in node metastases, since, at present, there is not a unique standard for their evaluation.

\section{Materials and Methods}

Surgical specimens for our retrospective study were selected among gastrectomies for gastric cancer performed between January 2008 and January 2011, at Desio, Carate Brianza and Giussano Hospitals (Italy), in patients who had not been treated with neoadjuvant therapy before. 34 gastric cancers with nodal metastases were chosen from this group and included in our study. All the samples were fixed with $10 \%$ buffered formalin and, afterwards, paraffin-embedded. For 
Table 1 ToGA trial criteria

\begin{tabular}{|c|c|c|}
\hline & Biopsy specimen staining pattern & Resection specimen staining pattern \\
\hline IHC 0 & No reactivity or no membranous reactivity in any tumor cell & $\begin{array}{l}\text { No reactivity or membranous reactivity in }<10 \% \text { of tumor } \\
\text { cells }\end{array}$ \\
\hline $\mathrm{IHC} 1+$ & $\begin{array}{l}\text { Tumor cell cluster with a faint or barely perceptible membranous } \\
\text { reactivity irrespective of percentage of tumor cells stained }\end{array}$ & $\begin{array}{l}\text { Faint or barely perceptible membranous reactivity in } \geq 10 \% \\
\text { of tumor cells; cells are reactive only in part of their } \\
\text { membrane }\end{array}$ \\
\hline IHC $2+$ & $\begin{array}{l}\text { Tumor cell cluster with a weak to moderate complete, basolateral or } \\
\text { lateral membranous reactivity irrespective of percentage of tumor } \\
\text { cells stained }\end{array}$ & $\begin{array}{l}\text { Weak to moderate complete, basolateral or lateral } \\
\text { membranous reactivity in } \geq 10 \% \text { of tumor cells }\end{array}$ \\
\hline IHC $3+$ & $\begin{array}{l}\text { Tumor cell cluster with a strong complete, basolateral or lateral } \\
\text { membranous reactivity irrespective of percentage of tumor cells } \\
\text { stained }\end{array}$ & $\begin{array}{l}\text { Strong complete, basolateral or lateral membranous reactivity } \\
\text { in } \geq 10 \% \text { of tumor cells }\end{array}$ \\
\hline
\end{tabular}

each case, three 3- $\mu$ m thick sections were cut from one primary tumor block, taking stock to select the most representative areas, avoiding necrotic and hemorrhagic spots; other three sections from the largest metastatic node were cut. One of these sections was prepared for hematoxylin-eosin staining, one section for IHC HER2 staining (a sample of IHC 3+ mammary tumor was put on each IHC glass as positive control) and, in IHC equivocal cases (2+), one further section was set up for Fluorescence In Situ Hybridization (FISH). When the IHC status of the primary tumor sample resulted negative, the test was repeated on other two representive blocks in order to avoid false negative result due to tumor heterogeneity. Instead, when the IHC score of the largest metastatic node was 3+, HER2 determination didn't go further, but in all the other cases, the test was repeated on all the other metastatic lymph nodes. IHC was performed by BenchMark XT immunostaining (Ventana, Tucson, Arizona) and HER2 was detected with the monoclonal antibody CB11 (Invitrogen Corporation, Camarillo, California). FISH was peformed using ZytoLight HER2/CEN17 Dual Color Probe Kit (ZytoVision GmbH, Bremerhaven, Germany). Three independent observers scored HER2 IHC status on each slide and, in case of disagreement, the case was jointly discussed (Cohen's $\mathrm{k}$ test was used for statistical analysis). All the observers were same-experienced in reading HER2 IHC slides and, purposely, no one was gastrointestinal or breast specialized pathologist: the aim of this choice was to obtain a standard assessment, comparable with the daily lab practice. HER2 expression was interpreted using criteria proposed by ToGA (Trastuzumab for GAstric cancer) trial (Table 1), which are suitable in biopsies and surgical specimens [2], and observing the three-steps "magnification rule" postulated by Rüschoff [3] The first step, which is evaluating each section at low magnification $(4 \mathrm{x})$, allows to decide for IHC $3+$ positivity. But if membranous reactivity was seen only at medium magnification (10-20x), the slide was scored as IHC $2+$. IHC $1+$ score needed high magnification $(40 \mathrm{x})$ to confirm membranous HER2 expression. Each metastasis case was scored using both endoscopic biopsy and resection specimen criteria. FISH was performed in all IHC 2+ cases. The total number of HER2 and chromosome 17 centromere (CEN17) control probe signals were counted in 20 interphase tumor cell nuclei and ratio of HER2 signals to CEN17 signals was calculated. When this ratio was $\geq 2.2$, the gene was considered to be amplified. In borderline cases, when ratio HER2/CEN17 was between 1.8 and 2.2, a group of other 20 nuclei was taken into consideration and the ratio was recalculated. Every case with IHC $3+$ score or FISH amplification is eligible for target therapy with trastuzumab.
Table 2 Tumors characteristics

\begin{tabular}{|c|c|}
\hline \multicolumn{2}{|c|}{ Lauren histological type } \\
\hline Intestinal & 19 \\
\hline Diffuse & 11 \\
\hline Mixed & 4 \\
\hline \multicolumn{2}{|l|}{ Site } \\
\hline Cardia & 6 \\
\hline Corpus & 11 \\
\hline Antrum & 17 \\
\hline \multicolumn{2}{|l|}{ pTNM } \\
\hline $\mathrm{T} 1 \mathrm{a}$ & 2 \\
\hline $\mathrm{T} 1 \mathrm{~b}$ & 1 \\
\hline $\mathrm{T} 2$ & 2 \\
\hline T3 & 15 \\
\hline $\mathrm{T} 4 \mathrm{a}$ & 11 \\
\hline $\mathrm{T} 4 \mathrm{~b}$ & 3 \\
\hline No & 0 \\
\hline N1 & 10 \\
\hline $\mathrm{N} 2$ & 10 \\
\hline N3 & 14 \\
\hline \multicolumn{2}{|l|}{ Stage } \\
\hline I & 3 \\
\hline II & 6 \\
\hline III & 25 \\
\hline IV & 0 \\
\hline
\end{tabular}




\section{Results}

The male female ratio was $0.89(16 \mathrm{M} / 18 \mathrm{~F})$; the mean age of the case study population was 68.5 years. Table 2 resumes tumors histopathologic features. Table 3 shows in the three first columns HER2 IHC scores in lymph node metastases, given by each of the 3 pathologists, and in the fourth column the score after collegial revision. A final column is dedicated to IHC scoring in corresponding primary tumors. Table 4 analytically defines the percentage agreement between single pathologists. The mean value was $71.4 \%(\kappa=0.45)$. Table 5 correlates HER2 status in primary tumor and metastatic site with Lauren histotypes. Comparative analysis between different scoring methods (biopsy vs resection) applied in lymph nodes revealed two discordant cases (\#4 and \#10). Case \#4 was evaluated IHC 2+ when using biopsy criteria and IHC 1+ with gastric resection scoring system. FISH revealed absence of amplification. Case \#10 was evaluated IHC $1+$ according to biopsy criteria and 0 according to resection criteria. In case $\# 1$, at first, the largest node was scored as positive (IHC $3+$ ) with biopsy criteria, whereas it resulted negative (IHC 0) applying resection criteria (Fig. 1). Then IHC was repeated on the other metastatic nodes (pN2) and we found other two completely positive
Table 3 Results of HER2 IHC scoring in metastases and in corresponding resection specimens
$x(B) / y(R)$ : different score of the same specimen by using biopsy criteria (B) and resection specimen criteria $(\mathrm{R})$

\begin{tabular}{|c|c|c|c|c|c|}
\hline & \multicolumn{4}{|l|}{ METASTASIS } & \multirow{2}{*}{$\begin{array}{l}\text { RESECTION } \\
\text { SPECIMEN }\end{array}$} \\
\hline & 1st pathologist & 2nd pathologist & 3rd pathologist & $\begin{array}{l}\text { After inter-pathologist } \\
\text { agreement }\end{array}$ & \\
\hline 1 & 3 & 3 & 3 & 3 & 0 \\
\hline 2 & 1 & 2 & 1 & 1 & 0 \\
\hline 3 & 1 & 2 & 1 & 1 & 0 \\
\hline 4 & $2(\mathrm{~B}) / 1(\mathrm{R})$ & 2 & 1 & 2 (B)/1 (R) & 0 \\
\hline 5 & 3 & 3 & 3 & 3 & 0 \\
\hline 6 & 0 & 0 & 0 & 0 & 0 \\
\hline 7 & 1 & 1 & 0 & 1 & 0 \\
\hline 8 & 1 & 1 & 1 & 1 & 1 \\
\hline 9 & 1 & 2 & 1 & 1 & 1 \\
\hline 10 & $1(\mathrm{~B}) / 0(\mathrm{R})$ & $1(\mathrm{~B}) / 0(\mathrm{R})$ & 1 & 1 (B)/0 (R) & 0 \\
\hline 11 & 1 & 2 & 1 & 1 & 1 \\
\hline 12 & 1 & 1 & 1 & 1 & 0 \\
\hline 13 & 2 & 2 & 1 & 2 & 0 \\
\hline 14 & 1 & 2 & 0 & 1 & 0 \\
\hline 15 & 1 & 2 & 0 & 1 & 1 \\
\hline 16 & 1 & 2 & 1 & 1 & 2 \\
\hline 17 & 1 & 1 & 1 & 1 & 1 \\
\hline 18 & 2 & 3 & 1 & 3 & 0 \\
\hline 19 & 2 & 2 & 1 & 2 & 0 \\
\hline 20 & 3 & 3 & 3 & 3 & 3 \\
\hline 21 & 3 & 3 & 2 & 3 & 3 \\
\hline 22 & 0 & 1 & 0 & 0 & 1 \\
\hline 23 & 0 & 0 & 0 & 0 & 1 \\
\hline 24 & 3 & 3 & 2 & 3 & 3 \\
\hline 25 & 3 & 3 & 2 & 3 & 3 \\
\hline 26 & 0 & 0 & 1 & 0 & 0 \\
\hline 27 & 1 & 1 & 1 & 1 & 1 \\
\hline 28 & 1 & 0 & 0 & 0 & 1 \\
\hline 29 & 0 & 0 & 0 & 0 & 0 \\
\hline 30 & 2 & 2 & 2 & 2 & 0 \\
\hline 31 & 0 & 0 & 0 & 0 & 0 \\
\hline 32 & 3 & 3 & 3 & 3 & 2 \\
\hline 33 & 0 & 0 & 0 & 0 & 1 \\
\hline 34 & 1 & 1 & 1 & 1 & 1 \\
\hline
\end{tabular}


Table 4 Inter-pathologists agreement

$$
\begin{aligned}
& \text { 1st vs 2nd pathologist } \\
& \text { Agreement }=82.48 \% \\
& \text { Cohen's } \kappa=0.61 \\
& \text { 2nd vs 3rd pathologist } \\
& \text { Agreement }=66.67 \% \\
& \text { Cohen's } \kappa=0.3 \\
& \text { 1st vs 3rd pathologist } \\
& \text { Agreement }=80.43 \% \\
& \text { Cohen's } \kappa=0.42
\end{aligned}
$$

$\kappa$ statistics interpretation for inter-rater reliability is: negative $K$, less than chance; $0.01<\kappa<0.2$, slight agreement; $0.21<\kappa<0.4$, fair agreement; $0.41<\mathrm{k}<0.6$, moderate; $0.61<\mathrm{k}<0.8$, substantial; $0.81<$ $\kappa<0.99$, basically perfect agreement areas, using both biopsy and gastric resection criteria (Fig. 2). In all other cases, the same result was found by applying both criteria. In every IHC $2+$ case, FISH analysis resulted negative, meaning that none of the cases showed HER2 amplification. The following step was to compare IHC lymph node metastasis score with the primary tumor one. Applying biopsy criteria, we observed concordance in 25 out of 34 cases $(73.5 \%$ ), while, using resection criteria, it was highlighted in 26 out of 34 cases (76.5\%). Six IHC 0 primary cancers were respectively assessed as IHC $2+$

\begin{tabular}{|c|c|c|c|c|c|}
\hline Case & Resection HER2 status & Histotype & Grade & Metastasis HER2 status & Metastatic pattern \\
\hline 1 & 0 & Int & G3 & 3 & Int \\
\hline 2 & 0 & Dif & G3 & 1 & Dif \\
\hline 3 & 0 & Int & G3 & 1 & Int \\
\hline 4 & 0 & Int & G3 & $2(\mathrm{~B}) / 1(\mathrm{R})$ & Int \\
\hline 5 & 0 & Mix & G3 & 3 & Mix \\
\hline 6 & 0 & Dif & G3 & 0 & Dif \\
\hline 7 & 0 & Dif & G3 & 1 & Dif \\
\hline 8 & 1 & Mix & G3 & 1 & Dif \\
\hline 9 & 1 & Int & G3 & 1 & Int \\
\hline 10 & 0 & Int & $\mathrm{G} 2$ & $1(\mathrm{~B}) / 0(\mathrm{R})$ & Int \\
\hline 11 & 1 & Int & G3 & 1 & Int \\
\hline 12 & 0 & Dif & G3 & 1 & Dif \\
\hline 13 & 0 & Dif & G3 & 2 & Dif \\
\hline 14 & 0 & Int & G2 & 1 & Int \\
\hline 15 & 1 & Int & G3 & 1 & Int \\
\hline 16 & 2 & Int & G2 & 1 & Int \\
\hline 17 & 1 & Dif & G3 & 1 & Dif \\
\hline 18 & 0 & Int & G3 & 3 & Int \\
\hline 19 & 0 & Mix & G3 & 2 & Mix \\
\hline 20 & 3 & Int & G3 & 3 & Int \\
\hline 21 & 3 & Int & G3 & 3 & Int \\
\hline 22 & 1 & Dif & G3 & 0 & Dif \\
\hline 23 & 1 & Dif & G3 & 0 & Dif \\
\hline 24 & 3 & Int & G3 & 3 & Int \\
\hline 25 & 3 & Int & $\mathrm{G} 2$ & 3 & Int \\
\hline 26 & 0 & Int & G3 & 0 & Int \\
\hline 27 & 1 & Int & G3 & 1 & Int \\
\hline 28 & 1 & Int & G3 & 0 & Int \\
\hline 29 & 0 & Dif & G3 & 0 & Dif \\
\hline 30 & 0 & Mix & G3 & 2 & Dif \\
\hline 31 & 0 & Int & G3 & 0 & Int \\
\hline 32 & 2 & Int & G3 & 3 & Int \\
\hline 33 & 1 & Dif & G3 & 0 & Dif \\
\hline 34 & 1 & Dif & G3 & 1 & Dif \\
\hline
\end{tabular}
(cases \#13 and \#19, \#30, FISH showed no amplification) and $3+$ (case \#1, \#5, \#18) after IHC analysis on lymph nodes. Cases \#16 and \#32, instead, resulted 2+ (FISH negatives) on surgical specimens, while they were respectively
Table 5 Comparison between HER2 status in primary tumor and metastatic site with histopathological features 


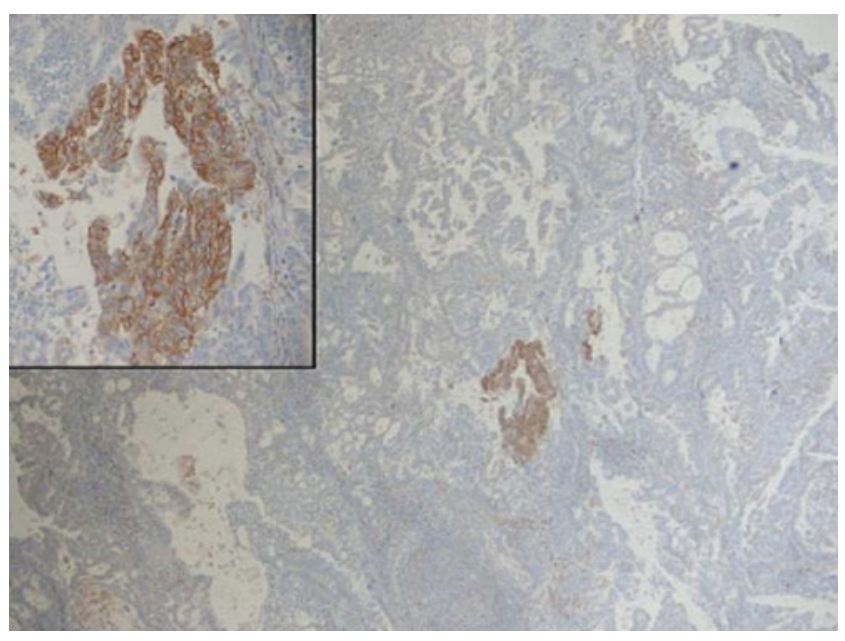

Fig. 1 A very interesting metastatic lymph node section (case \#1) in which only a small cluster of tumor cells expresses membranous reactivity for HER2. This case could be classified as $3+$ by using biopsy criteria, and as 0 if we adopt gastrectomy criteria

scored as $1+$ and $3+$ on node metastasis. Case \#4 was declared HER2 negative (IHC 0 ) on the primary tumor site, while, only applying the biopsy criteria we observed a discordant status in the metastatic node (IHC 2+, FISH negative).

Table 6 defines the percentage distribution of HER2 IHC scores in our cases by using biopsy specimen criteria and resection specimen criteria. Applying biopsy criteria we observed 22 negative cases for HER2 protein expression (64.7\%), 4 borderline cases (11.8\%) and 8 positive cases $(23.5 \%)$, whereas the use of resection criteria showed 23 negative $(67.7 \%), 3$ borderline $(8.8 \%)$ and 8 positive cases $(23.5 \%)$.

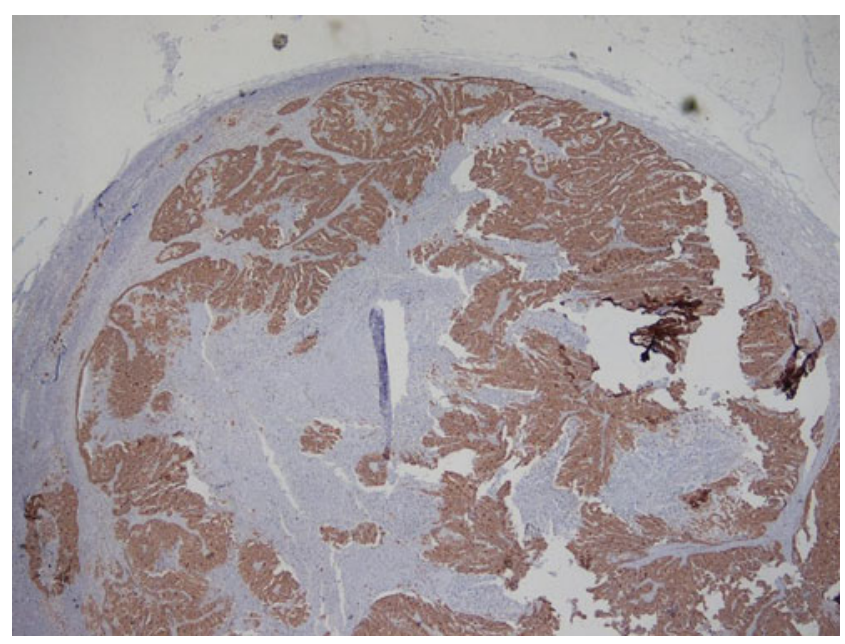

Fig. 2 Another lymph node from case \#1 with a clearly IHC 3+ metastatic localization
Table 6 Distribution of HER2 IHC scores in our cases by using biopsy specimen criteria and resection specimen criteria

\begin{tabular}{lcc}
\hline Score IHC & $\begin{array}{l}\text { With biopsy specimen } \\
\text { criteria } n(\%)\end{array}$ & $\begin{array}{l}\text { With resection specimen } \\
\text { criteria } n(\%)\end{array}$ \\
\hline 0 & $8(23.5)$ & $9(26.5)$ \\
$1+$ & $14(41.2)$ & $14(41.2)$ \\
$2+$ & $4(11.8)$ & $3(8.8)$ \\
$3+$ & $8(23.5)$ & $8(23.5)$ \\
\hline
\end{tabular}

\section{Discussion}

The global frequency of HER2 gene amplification in gastric cancer is between 7.1 and $42 \%$, while IHC protein overexpression is between 6.8 and $34.9 \%$ [2]. This broadness highlights the lack of a high-reproducible reporting system. The availability of extensive lymph node dissections derived from gastrectomies for gastric cancer provides with an excellent opportunity to test HER2 IHC and FISH and to study diagnostic protocols. The determination of HER2 IHC status in gastric carcinoma is harder than in breast carcinoma, because of peculiar features of this tumor. First of all, the finding of incomplete immunoreactive membranes (socalled "U" shape) is very common, because of high frequency of glandular formations. Thus, cells with basolateral membrane reactivity should be considered identical to those with complete membranous reactivity. Moreover, HER2 expression is very heterogeneous and it is common to find alternation, within the same tumor, of areas with moderate to strong reactivity and areas without reactivity. Literature has therefore defined different interpretation criteria for biopsy and resection specimen [1]. In biopsies, indeed, a tight cluster of 5 immunoreactive cells is enough to define HER2 positivity, while in resection specimens membranous reactivity is to be found in $10 \%$ or more of tumor cells [4]. Preliminary data [5] report that HER2 status assessed in resection specimens is generally maintained even in metastatic cells, which are the main target of therapy in advanced disease, because they should be a selection of cell clones with greater invasive abilities. Despite their importance in terms of prognosis, up to now there is not a standardized evaluation system to graduate IHC reactivity in lymph node metastases. As said before, one of the aims of this study is to consider the differences that could emerge from application of different diagnostic protocols in the assessment of HER2 IHC status in lymph nodes metastasis. We must underline that a major limitation of our study is the number of cases. Our purpose was only to suggest a debate in the pathologist community, but surely multi-institutional and prospective studies with larger populations could clarify this issue and obtain more realistic data. 


\section{Expected HER2 Positive Metastases Percentages}

The distribution of HER2 positivity in our study is consistent with few available data from literature and this is necessary in order to prove our correct application of IHC HER2 status assessement criteria. For example, Bozzetti et al. obtained 22 negative cases $(56.4 \%), 10$ borderline $(25.6 \%)$ and 7 positive (18\%) in a record of 39 gastric adenocarcinoma metastases [6]. Moreover, this agreement confirms that the tumor population of gastric adenocarcinomas presented in this paper is qualitatively similar to the one of other study groups.

\section{HER2 Status IHC Examination Inter-observer Variability}

The mean value of inter-pathologist agreement was $71.4 \%$, which is in line with data presented in literature [7]. Interobserver variability in HER2 status assessment was not insignificant (percentage agreement was, respectively, $75 \%$ between 1 st and 2 nd observer, $53.1 \%$ between 2nd and 3rd and $78.1 \%$ between 1 st and 3rd): for this reason, according to our experience, we suggest to consider collegial discussion as the best way to decrease inter-observer disagreement in the most equivocal cases. This precaution, which is fundamental in gastric adenocarcinoma biopsy specimens, should be applied also in nodal metastases.

\section{HER2 Status Variability Between Primary Tumor (gastric resection) and Nodal Metastasis}

In our study, we found a significant HER2 expression variability between lymph node metastasis and primary tumor, and this evidence reflects the concept of intratumoral heterogeneity. Despite preliminary studies [8] report that gastric carcinomas do not show significant HER2 status changes from primary tumor to metastatic sites, we observed significant difference in 9 cases (with biopsy criteria) of our record. Because of the small sample of this study, we cannot provide a satisfactory answer about this topic. Anyway, our results highlight the heterogeneity and the biological complexity of gastric tumor and stress the importance of a reproducible and analytical standardized system, which clearly defines eligibility criteria to the target therapy.

\section{Applying Different Scoring Systems Leads to Different} Assessment

ToGA trial defined two different scoring systems for primary tumor, respectively for endoscopical biopsies and surgical specimens, but it did not suggest a diagnostic protocol for node metastases. Thus, we are wondering what kind of criteria (biopsy vs resection) is the most suitable for HER2 IHC status assessment in lymph nodes. With the limits we have discussed above, our study doesn't identify a single patient who would have undergone a different treatment, depending on the scoring criteria. However, for case \#1, the first analyzed node, according to resection criteria, would have been scored as IHC 0 , not allowing the patient to be eligible for target therapy (moreover, the primary tumor was IHC 0). On the other hand, the biopsy criteria permits to score this nodal metastases as IHC $3+$. The evaluation of every metastatic node interestingly revealed two other massively metastatic lymph nodes with diffuse immunoreactivity (IHC $3+$ with both the criteria).

Possible Importance of Testing HER2 in Multiple Metastatic Nodes to Avoid False Negatives Results Due to Tumor Heterogeneity

In our report we detected some cases (also \#5) which stressed the importance of testing HER2 in multiple metastatic nodes when the primary tumor and the largest node are negative. In fact, only a complete evaluation of all the lymph nodes permitted to reveal two intestinal pattern metastatic areas with strong membranous immunoreactivity, virtually enabling the use of trastuzumab for this patient.

\section{References}

1. Bang YJ, Van Cutsem E, Feyereislova A, Chung HC, Shen L, Sawaki A, Lordick F, Ohtsu A, Omuro Y, Satoh T, Aprile G, Kulikov E, Hill J, Lehle M, Rüschoff J, Kang YK (2010) Trastuzumab in combination with chemotherapy versus chemotherapy alone for treatment of HER2-positive advanced gastric or gastro-oesophageal junction cancer (ToGA): a phase III, openlabel, randomised controlled trial. Lancet 376:687-97

2. Hoffmann M, Stoss O, Shi D, Buttner R, van de Vijver M, Kim W, Ochiai A, Rüschoff J, Henkel T (2008) Assessment of a HER2 scoring system for gastric cancer: results from a validation study. Histopathology 52:797-805

3. Rüschoff J, Dietel M, Baretton G, Arbogast S, Walch A, Monges G, Chenard MP, Penault-Llorca F, Nagelmeier I, Schlake W, Höfler H, Kreipe HH (2010) HER2 diagnostics in gastric cancer: guideline validation and development of standardized immunohistochemical testing. Virchows Arch 457:299-307

4. Wolff AC, Hammond EH M, Schwartz JN, Hagerty KL, Allred DC, Cote RJ, Dowsett M, Fitzgibbons PL, Hanna WM, Langer A, McShane LM, Paik S, Pegram MD, Perez EA, Press MF, Rhodes A, Sturgeon C, Taube SE, Tubbs R, Vance GH, van de Vijver M, Wheeler TM, Hayes DF (2007) American Society of Clinical Oncology/College of American Pathologists Guideline recommendations for Human Epidermal Growth Factor Receptor 2 testing in breast cancer. Arch Pathol Lab Med 131:18-43

5. Albarello L, Pecciarini L, Doglioni C (2011) HER2 Testing in gastric cancer. Adv Anat Pathol 18:53-59

6. Bozzetti C, Negri FV, Lagrasta CA, Crafa P, Bassano C, Tamagnini I, Gardini G, Nizzoli R, Leonardi F, Gasparro D, Camisa R, Cavalli S, Silini EM, Ardizzoni A (2011) Comparison of HER2 status in 
primary and paired metastatic sites of gastric carcinoma. Br J Cancer 104:1372-1376

7. Nassar A, Cohen C, Agersborg SS, Zhou W, Lynch KA, Albitar M, Barker EA, Vanderbilt BL, Thompson J, Heyman ER, Lange H, Olson A, Siddiqui MT (2001) Trainable Immunohistochemical HER/neu Image Analysis. A multisite performance study using 260 breast tissue specimen. Arch Pathol Lab Med 135:896-902

8. Marx AH, Tharun L, Muth J, Dancau AM, Simon R, Yekebas E, Kaifi JT, Mirlacher M, Brümmendorf TH, Bokemeyer C, Izbicki JR, Sauter G (2009) HER-2 amplification is highly homogeneous in gastric cancer. Hum Pathol 40:769-777

\section{Ethical Note}

In our study HER 2 positivity was found in 8 out of 34 patients. One of these underwent surgery after edition of ToGA trial, so he was able to receive the biological drug in addiction to conventional chemotherapy regimen, on oncologists' recommendation. The other 7 patients were operated before August 2010, so they did not receive eligibility indication for Trastuzumab. After our study, the diagnosis of HER2 positivity in primary tumor or in metastatic site was communicated to oncologists in order to take in consideration the option of a further specific treatment. 